\title{
UM MODELO FUZZY COMPORTAMENTAL PARA ANÁLISE DE SOBRE-REAÇÃO E SUB-REAÇÃO NO MERCADO DE AÇÕES BRASILEIRO
}

A FUZZY BEHAVIORAL MODEL FOR ANALYZING OVER-REACTION AND UNDER-REACTION IN THE BRAZILIAN STOCK MARKET

\section{RESUMO}

Neste artigo, são apresentados testes empíricos para a investigação de ocorrência de fenômenos de sobre-reação e sub-reação no mercado de ações brasileiro. Para esses testes, é proposto um modelo baseado na teoria de conjuntos Fuzzy, que possui forte relação com as heurísticas de representatividade e ancoramento, estabelecidas na teoria de finanças comportamentais. O modelo proposto é empregado para a formação de carteiras e utiliza indicadores financeiros de companhias abertas. Para as análises são utilizados dois conjuntos de ações, um do setor de petróleo e petroquímica e outro do setor têxtil, com indicadores financeiros relativos ao período de 1994 a 2005.

\section{Renato Aparecido Aguiar}

Professor do Departamento de Engenheira Elétrica, Centro Universitário da Fundação Educacional Inaciana renato.aguiar@poli.usp.br

\section{Roberto Moura Sales}

Professor do Departamento de Engenharia de Telecomunicações e Controle da Escola Politécnica, Universidade de São Paulo roberto@lac.usp.br

\section{Lucy Aparecida de Sousa}

Professora do Departamento de Economia, Fundação Armando Álvares Penteado

lucy@interlinkconsultoria.com.br

Recebido em 21.02.2006. Aprovado em 25.06.2007

Avaliado pelo sistema double blind review

Editor Científico: Ricardo Ratner Rochman

\begin{abstract}
This paper presents empirical tests for investigating the occurrence of over-reaction and under-reaction phenomena in the Brazilian stock market. It is proposed that a model based on the theory of fuzzy sets, which bears a strong relationship to representativeness and anchoring heuristics, as established in the theory of behavioral finance, should be used for these tests. The proposed model is used to form portfolios and uses the financial indicators of publicly-quoted companies. Two lots of shares were used for the analyses; one from the oil and petro-chemical sector and the other from the textile sector; the financial indicators for the period 1994 to 2005 were used.
\end{abstract}

PALAVRAS-CHAVE Sobre-reação, sub-reação, conjuntos fuzzy, finanças comportamentais, classificação de ações KEYWORDS Over-reaction, under-reaction, fuzzy sets, behavioral finance, share classification. 


\section{INTRODUÇÃO}

Há décadas o desenvolvimento de ferramentas que auxiliam na tomada de decisões de investidores na área de finanças tem sido objeto de intensa pesquisa em todo o mundo. De modo geral, as decisões financeiras visam à maximização de retornos futuros dos ativos, mas, com a evolução dessa e de outras premissas básicas, produziram-se teorias conflitantes, como é o caso da Teoria de Mercados Eficientes e a Teoria de Finanças Comportamentais (SHILLER, 2002). Cada teoria estruturou-se a partir de contribuições fundamentais de um grande número de autores (MODIGLIANI e MILLER, 1958; SHARPE, 1964; FAMA, 1970; BARBERIS, SHLEIFER e VISHNY, 1998; PETERS, 2003; PENNINGS, 2003; BENZION e YAGIL, 2003; RITTER, 2003), incluindo trabalhos que receberam o prêmio Nobel em Economia, como a Teoria de Portfólio, proposta por Markowitz (1952, 1959), e a Teoria de Finanças Comportamentais, proposta por Kahnemann (2002) e Smith (2002).

Em linhas gerais, a Teoria de Mercados Eficientes pressupõe que o investidor é racional e avesso ao risco. Conseqüentemente, as decisões dos investidores são tomadas com base em informações estatísticas e probabilidades que projetam o desempenho futuro dos ativos e de todo o mercado financeiro. Em outra abordagem, a Teoria Comportamental considera fatores de caráter psicológico dos investidores na tomada de decisões, ou seja, supõe que os investidores não são totalmente racionais e usam heurísticas e regras práticas e que suas decisões são afetadas por preferências e crenças. Alguns dos exemplos citados como violação da racionalidade, baseando-se na primeira teoria, são a pouca aversão ao risco, o excesso de confiança e a sub ou sobre-reação às informações. Os artigos de Shiller (2002), Lima (2003) e Kimura (2003) apresentam uma ampla exposição de questionamentos a essas duas teorias.

Apesar das diferenças de opiniões, as finanças comportamentais têm-se mostrado uma ferramenta adequada na abordagem de diversos problemas. $\mathrm{Na}$ área de precificação de ativos, por exemplo, tem sido usada para interpretar fenômenos que envolvem retornos de ativos. Da análise de informações relativas ao comportamento do mercado financeiro, tem-se aprendido muito sobre a conduta de investidores e analistas. $\mathrm{Na}$ área de finança corporativa, a abordagem comportamental tem alertado para atitudes tais como excessiva aversão a risco e excessivo otimismo (KAHNEMANN, 2002).

Com base nessa abordagem, o presente trabalho realiza um estudo de fenômenos de sobre-reação e sub-reação relacionados ao mercado de ações brasileiro, tendo como intuito investigar a viabilidade de estratégias operacionais classificadas como estratégias de momento e estratégias contrárias.

Estudos de problemas semelhantes em diferentes mercados (DeBONDT e THALER, 1985), realizados no mercado dos Estados Unidos, constataram que, quando ações são ranqueadas com base nos retornos passados de três a cinco anos, vencedores passados tendem a ser perdedores futuros, e vice-versa. Este efeito foi atribuído à sobre-reação do investidor. Através de uma análise de retornos de ativos no mercado chinês, Kang e outros (2002) encontraram sobre-reação a curto prazo e sub-reação a médio prazo, estatisticamente significantes.

Outros trabalhos que também abordam o problema de sobre-reação e sub-reação, utilizando conceitos de finanças comportamentais, são os de Jegadeesh e Titman (1993), que identificam a ocorrência de sub-reação ao constatar que, a médio prazo, vencedores passados continuam a superar perdedores passados; de Jegadeesh (1990), que constatam sobre-reação relativa a um horizonte de curto prazo; de Kimura (2003), que adapta o modelo desenvolvido por DeBondt e Thaler ao mercado de ações do Brasil; de Minardi (2004), que investiga se séries históricas de preços podem prever retornos futuros, utilizando uma metodologia análoga à de Jegadeesh (1990) e Barberis, Shleifer e Vishny (1998); e, finalmente, os estudos de Daniel, Hirshleifer e Subrahmanyam (1998), que explicam como os vieses de julgamento dos investidores podem produzir sobre-reação ou sub-reação em função de alguns eventos.

Para os desenvolvimentos aqui apresentados, avaliase o elo, ainda pouco investigado na literatura, conforme observado em Peters (1996), entre a teoria de conjuntos Fuzzy, originalmente proposta por Zadeh (1965), e as heurísticas da teoria de finanças comportamentais. $\mathrm{O}$ objetivo é, assim, identificar o índice de pertinência associado a diferentes conjuntos de ações e avaliar certas propriedades de cada um desses conjuntos no mercado financeiro. Para isso, desenvolve-se uma metodologia, utilizando-se o algoritmo denominado Fuzzy Clustering Means, para a classificação desse conjunto de ações do mercado brasileiro, a partir de índices financeiros das empresas correspondentes. Para verificação empírica, são utilizados dois conjuntos de ações: ações do setor de petróleo e petroquímica e ações do setor têxtil, referentes ao período de 1994 a 2005. Mostra-se, então, que o Modelo Fuzzy Comportamental proposto incorpora influências heurísticas da teoria comportamental; a partir do modelo para classificação de ações, são apresentados testes para as hipóteses de sobre-reação e sub-reação. 
O trabalho está organizado da seguinte maneira. $\mathrm{Na}$ primeira seção, são apresentados conceitos sobre finanças comportamentais e modelos baseados nessa teoria. Na segunda seção, são apresentados conceitos básicos relativos a conjuntos Fuzzy e o algoritmo Fuzzy Clustering Means. A metodologia para obtenção do Modelo Fuzzy Comportamental proposto é apresentada na seção seguinte. Na quarta seção, explicita-se o procedimento para a realização dos testes empíricos de hipóteses de sub-reação e sobre-reação. Os resultados são apresentados na quinta seção. Finalmente, encaminha-se a conclusão, apontando as contribuições deste estudo e os seus limites.

\section{FINANÇAS COMPORTAMENTAIS}

No início da década de 1970, quando a teoria de mercados eficientes já havia atingido um alto grau de influência no pensamento econômico, a busca do entendimento de anomalias de comportamento em mercados financeiros em todo o mundo trouxe a inclusão de conceitos da psicologia e da sociologia nas análises econômicas, a ponto dos trabalhos de Kahnemann (2002) e Smith (2002) terem sido merecedores do prêmio Nobel de Economia em 2002. Os elementos envolvidos nessa nova abordagem levaram à elaboração da chamada Teoria de Finanças Comportamentais.

Em contraposição à teoria de mercados eficientes, a teoria comportamental assume que indivíduos tomam decisões governados por heurísticas, ou regras práticas, baseados em raciocínio que se desvia de regras estatísticas. A psicologia cognitiva, que estuda o processo mental hipoteticamente por detrás do comportamento, está na base dessa abordagem e mostra que os indivíduos valorizam excessivamente as experiências recentes e são demasiadamente confiantes em suas próprias habilidades, o que leva a distorções em seu pensamento (RITTER, 2003).

São diversas as heurísticas que influenciam a tomada de decisões. A seção seguinte descreve o viés produzido pelo uso das heurísticas de representatividade e de ancoramento (PETERS, 1996), as quais estão diretamente relacionadas com a teoria de conjuntos Fuzzy, conforme tratado na seção seguinte.

\section{Heurística de Representatividade}

A heurística de representatividade está associada essencialmente à similaridade entre os elementos estudados. A seguir, é apresentado um exemplo clássico proposto por Peters (2003), em que a tomada de decisão, mesmo quando é conhecida a probabilidade de ocorrência dos resultados, é fortemente influenciada por informações descritivas, isto é, os indivíduos preferem valorizar informações descritivas a considerar as probabilidades de ocorrência do fato.

No exemplo, indivíduos devem responder qual a ocupação de uma pessoa escolhida aleatoriamente em um grupo de dez, sabendo-se que oito pessoas do grupo são motoristas de caminhão e duas são corretores. No primeiro procedimento, as dez pessoas estão igualmente vestidas e, após a escolha de uma dentre as dez, a maioria dos participantes, apoiada na probabilidade conhecida, julgaram que esta pessoa seria um motorista de caminhão. Já no segundo procedimento foi adicionado um elemento de ambigüidade; as dez pessoas foram vestidas diferentemente e foi escolhida uma pessoa vestindo terno, óculos e portando uma pasta. Nesse caso, a maioria dos participantes identificou o escolhido como corretor, apesar da probabilidade de ele ser motorista de caminhão superar a probabilidade, conhecida a priori, de ser corretor.

Nesse exemplo, o homem vestindo terno, óculos e portando uma pasta tem mais similaridade com o conjunto de corretores e menos similaridade com o conjunto de motoristas de caminhão. Os indivíduos que se baseiam em informações descritivas disponíveis tornam-se mais confiantes quando surge uma nova informação, representada pelo elemento de ambigüidade e, no exemplo anterior, respondem que a pessoa selecionada era um corretor (PETERS, 1996).

No contexto das decisões em economia, um indivíduo sob a influência da heurística de representatividade tem uma forte tendência a dar mais ênfase às informações recentes. Como no exemplo anterior, o fato de haver uma nova informação ambígua reduz a precisão da análise, produzindo decisões com viés de sobre-reação (AMIR e GANZACH, 1998).

No caso específico do mercado de ações, a hipótese de sobre-reação estabelece que, quando o investidor não está seguro em relação ao preço intrínseco de uma ação, há uma tendência em dar mais ênfase às notícias recentes associadas à ação, em detrimento de sua crença passada ou de informações passadas. Dessa forma, o investidor sob a influência da heurística de representatividade tem uma reação excessivamente otimista (pessimista) quando o valor de uma ação está aumentando (diminuindo) (OFFERMAN e SONNEMANS, 2004; BAYTAS e CAKICI, 1999).

Experimentos desse tipo fortalecem a hipótese de que, 
sob condições de ambigüidade, um método que utiliza conjuntos Fuzzy é mais adequado para modelar a tomada de decisão do que métodos estatísticos, visto que a tomada de decisão sob condições de ambigüidade ancora-se na similaridade, ignorando a probabilidade de ocorrência (PETERS, 2003).

\section{Heurística de Ancoramento}

Freqüentemente, as pessoas baseiam-se em elementos ou condições de referência para tomar decisões. Nesse caso, diz-se que a decisão está ancorada em um referencial, ou seja, a decisão baseia-se em uma "heurística de ancoramento". A heurística de ancoramento, diferentemente da "heurística de representatividade", conduz ao excesso de moderação na tomada de decisão, produzindo assim o fenômeno de sub-reação (AMIR e GANZACH, 1998), em que vencedores passados tendem a ser vencedores futuros e perdedores passados tendem a ser perdedores futuros (FAMA, 1998). A heurística de ancoramento está associada a decisões conservadoras, fazendo com que as pessoas resistam a mudanças bruscas de suas decisões quando se deparam com novas informações.

Em termos da teoria de conjuntos Fuzzy, como veremos a seguir, uma decisão com base nesta heurística é focada no elemento de maior referência no conjunto, ou seja, o elemento de pertinência total, $\mu(\mathrm{x})=1$ (PETERS, 1996).

\section{Modelos em Finanças Comportamentais}

Conforme já mencionado, a teoria de finanças comportamentais incorpora elementos diferentes daqueles considerados na teoria de mercados eficientes (RITTER, 2003). Esse fato tem permitido o desenvolvimento de alguns novos modelos para explicar possíveis anomalias no mercado financeiro. Tais anomalias, quando inexplicáveis com as ferramentas clássicas, têm encontrado explicações satisfatórias através da teoria de finanças comportamentais (STRACCA, 2004). A seguir, são citados alguns modelos baseados nas heurísticas de representatividade e ancoramento.

\section{Modelo de Barberis, Shleifer e Vishny}

No trabalho desenvolvido por Barberis, Shleifer e Vishny (1998), foi proposto um modelo do sentimento do investidor baseado em viés comportamental decorrente das heurísticas citadas, o qual induz investidores a subreagir ou sobre-reagir às informações disponíveis. Nesse modelo, os lucros seguem um passeio aleatório, porém os investidores crêem que o comportamento dos lucros se dá em dois regimes. No regime 1, os lucros revertem à média, enquanto no regime 2 , os lucros seguem certa tendência.

Quando os investidores acreditam que o regime 1 atua no mercado, os preços das ações sub-reagem à mudanças nos lucros, pois o investidor reage muito pouco aos anúncios de alterações recentes nos lucros. Por outro lado, os investidores extrapolam incorretamente a tendência quando acreditam que o regime 2 atua no mercado, fazendo com que o preço da ação tenda a sobre-reagir.

Modelo de Daniel, Hirshleifer e Subrahmanyam O modelo de Daniel, Hirshleifer e Subrahmanyam (1998) visa conciliar as constatações empíricas de sobre-reação e sub-reação. Segundo esses autores, os investidores sem informação não apresentam viés comportamental, enquanto os investidores informados são influenciados por dois tipos de viés: o excesso de confiança, que está associado à heurística de representatividade, e a valorização demasiada de suas percepções, associada à heurística de ancoramento. A valorização demasiada da percepção conduz os investidores a darem menos ênfase às avaliações disponíveis publicamente, exagerando a precisão de suas análises sobre os valores dos ativos. Como conseqüência, os autores constatam que a sobrevalorização da informação privada e a sub-valorização da informação pública tendem a produzir sub-reação a curto prazo, seguida de sobre-reação a longo prazo, quando a informação pública tende a superar o viés comportamental.

\section{Modelo de Hong e Stein}

No modelo de Hong e Stein (1999), são caracterizados dois tipos de agentes: os espectadores de notícias e os operadores de momento. Os espectadores de notícias tomam decisões a partir de idéias e opiniões próprias, desprezando informações correntes e passadas. Os operadores de momento condicionam suas análises a medidas de preços passados, não levando em conta novas informações.

Nesse modelo, assumindo inicialmente a tendência à sub-reação, constata-se que, com a inclusão de operadores de momento no mercado, cria-se uma elevação excessiva nos preços dos ativos, gerando, dessa forma, uma sobrereação. Assim, pelo modelo de Hong e Stein, a existência de sub-reação pode gerar a sobre-reação.

Algumas pesquisas similares têm sido feitas em outros mercados, com adaptação de metodologias já existentes. Como exemplo, Kang e outros (2002), baseados no método proposto por Jegadeesh e Titman (1993), avaliam 
a existência de sub-reação ou sobre-reação no mercado de ações da China. Kimura (2003) adapta a metodologia apresentada em DeBondt e Thaler (1985) ao mercado de ações do Brasil para investigar a influência de viés comportamental nos preços das ações. Também para ações no Brasil, Minardi (2004) verifica se séries históricas de preços de ações são capazes de prever retornos futuros, utilizando uma metodologia análoga à de Jegadeesh (1990).

\section{TEORIA DOS CONJUNTOS FUZZY E BASE MATEMÁTICA}

A teoria de conjuntos Fuzzy foi originalmente apresentada por Zadeh (1965) e tem, como uma de suas principais características, o fato de permitir o tratamento de problemas que envolvam variáveis chamadas lingüísticas, tais como: quente, muito quente, alto, baixo, recomendável, pouco recomendável, muito arriscado etc.

A propriedade que surge quando se consideram variáveis lingüísticas para caracterizar objetos estudados é que, ao invés de esses objetos pertencerem ou não a um certo conjunto - como determina a teoria clássica de conjuntos, seus índices de pertinência estarão associados a diferentes conjuntos.

Como exemplo, pode-se dizer que um brasileiro com $1,80 \mathrm{~m}$ de altura tem um índice de pertinência maior associado ao conjunto de pessoas altas do que o índice de pertinência associado ao conjunto de pessoas baixas. Dito de outra maneira, essa pessoa tem um perfil que mais se aproxima de uma pessoa alta do que de uma pessoa baixa.

Nas aplicações, a teoria Fuzzy tem sido empregada em diversas áreas, como na engenharia (GRAHAM e NEWELL, 1989), na medicina (BATES e YOUNG, 2003), na biologia (WOOLF e WANG, 2000) e também na economia (DOURRA e SIY, 2002; WONG e WANG, 1991). Para Zadeh (1973), os inúmeros exemplos apresentados na literatura mostram que o emprego de variáveis lingüísticas e algoritmos Fuzzy constitui uma ferramenta poderosa para a modelagem e análise do comportamento de sistemas complexos, que apresentem fenômenos cuja descrição matemática não possa ser feita de forma adequada através de equações algébricas ou diferenciais.

\section{Conjuntos Fuzzy}

Nesta seção, são apresentados conceitos básicos da teoria de conjuntos Fuzzy. Como essa teoria possui várias ramificações, que incluem diversos conceitos além dos necessários para o entendimento do algoritmo Fuzzy Clustering Means aqui utilizado, a apresentação a seguir está organizada de forma delimitada. Isso não deve ser interpretado, porém, como um desestímulo para o aprofundamento dessa teoria, uma vez que nossa opinião é de que a pesquisa nesse campo poderá trazer importantes desenvolvimentos futuros. Existem diversos textos clássicos que podem ser consultados para compreensão mais profunda acerca da teoria Fuzzy (ZADEH, 1965, 1973; ZIMMERMANN, 1996; DIAMOND e KLOEDEN, 1994 KAUFMANN, 1975).

Nessa teoria, o índice de pertinência pode ser entendido como uma medida do grau de afinidade, similaridade ou compatibilidade entre elementos.

Definição 1: Sejam o conjunto $X=\left\{\mathrm{x}_{1}, \mathrm{x}_{2}, \ldots, \mathrm{x}_{\mathrm{m}}\right\}$ e $\mathrm{C}_{1}$, $\mathrm{C}_{2}, \ldots, \mathrm{C}_{\mathrm{n}}$ subconjuntos de $\mathrm{X}$. Sejam ainda números reais $0 \leq \mu_{\mathrm{i}}\left(\mathrm{x}_{\mathrm{j}}\right) \leq 1, \mathrm{i}=1,2, \ldots, \mathrm{n}$ e $\mathrm{j}=1,2, \ldots, \mathrm{m}$, tais que, para todo $\mathrm{j}=1,2, \ldots, \mathrm{m}$, tem-se $\sum^{\mathrm{n}} \mu_{\mathrm{i}}\left(\mathrm{x}_{\mathrm{j}}\right)=1$. Nessas condições, $\mu_{\mathrm{i}}\left(\mathrm{x}_{\mathrm{j}}\right)$ é chamado índice de pertinência do elemento $x_{j}$ com relação ao subconjunto $C_{i}$. $O$ exemplo seguinte ilustra o significado desta definição.

Exemplo 1: Seja o conjunto

$X=\left\{x_{1}=(1,2), x_{2}=(2,1), x_{3}=(2,3), x_{4}=(4,1), x_{5}=(4,3)\right.$, $\left.\mathrm{x}_{6}=(5,2),\right\}$ e os subconjuntos $C_{1}$ e $C_{2}$ representados na Figura 1 .

Segundo a teoria clássica de conjuntos, ao escrever $C_{1}=\left\{x_{1}, x_{2}, x_{3}\right\}$ e $C_{2}=\left\{x_{4}, x_{5}, x_{6}\right\}$, fica estabelecido que

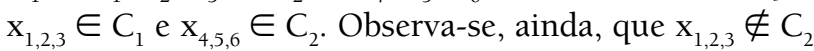
e $\mathrm{x}_{4,5,6} \notin \mathrm{C}_{1}$. No contexto da teoria de conjuntos Fuzzy, porém, a associação de índices de pertinência aos elementos $\mathrm{x}_{\mathrm{i}}$ introduz a idéia de que $\mathrm{x}_{1}, \mathrm{x}_{2}$ e $\mathrm{x}_{3}$ "pertencem mais" a $C_{1}$ do que pertencem a $C_{2}$ e $x_{4}, x_{5}$ e $x_{6}$ "pertencem mais" a $C_{2}$ do que pertencem a $C_{1}$. Embora esses índices possam ser definidos de várias formas, uma definição bastante simples, para esse exemplo, é $\mu_{1}\left(\mathrm{x}_{1}\right)=\mu_{2}\left(\mathrm{x}_{6}\right)=1 \mathrm{e}$ $\mu_{2}\left(x_{1}\right)=\mu_{1}\left(x_{6}\right)=1$, indicando pertinência total de $x_{1} e m$ relação a $C_{1}$, pertinência nula de $x_{1} e m$ relação a $C_{2}$, pertinência total de $x_{6}$ em relação a $C_{2}$ e pertinência nula de $\mathrm{x}_{6}$ em relação a $C_{1}$. Claramente, essa definição satisfaz às condições $\mu_{1}\left(x_{1}\right)+\mu_{2}\left(x_{1}\right)=1$ e $\mu_{1}\left(x_{6}\right)+\mu_{2}\left(x_{6}\right)=1$. Para os demais elementos, os índices de pertinência poderiam ser definidos, por exemplo, com base nas distâncias em relação aos elementos $x_{1}$ e $x_{6}$, respectivamente, que são os elementos de pertinência total. A fim de respeitar as condições $\sum_{\mathrm{i}=1}^{2} \mu_{\mathrm{i}}\left(\mathrm{x}_{\mathrm{j}}\right)=1, \mathrm{j}=2,3,4,5$, essas distâncias devem, 
no entanto, ser normalizadas pela soma das distâncias em relação a esses pontos de pertinência total. Ainda, de forma a se ter maior pertinência quanto menor for a distância, faz-se
Algoritmo Fuzzy Clustering Means (FCM)

Dentre as técnicas para o agrupamento ou a classificação de elementos em subconjuntos de um conjunto dado, o algoritmo Fuzzy Clustering Means (FCM) constitui uma ferramenta eficiente nos casos em que as características ou os atributos dos elementos estudados podem ser representados através de um vetor de números reais. Nesses casos, com base em uma matriz denominada matriz-padrão de dimensão $\mathrm{n} \times \mathrm{p}$, sendo $\mathrm{n}$ o número de elementos $\mathrm{e} p$ a dimensão dos vetores das características desses elementos, o algoritmo FCM permite identificar clusters ou agrupamentos de elementos, tais que os elementos de um mesmo grupo sejam mais similares
Analogamente,

$\mu_{1}\left(x_{3}\right)=1-\frac{d\left(x_{3}, x_{1}\right)}{d\left(x_{3}, x_{1}\right)+d\left(x_{3}, x_{6}\right)}=0,691$
$\mu_{2}\left(x_{3}\right)=1-\frac{d\left(x_{3}, x_{6}\right)}{d\left(x_{3}, x_{1}\right)+d\left(x_{3}, x_{6}\right)}=0,309$
$\mu_{1}\left(x_{4}\right)=0,309 \quad \mu_{2}\left(x_{4}\right)=0,691$

ou mais compatíveis, que elementos em grupos diferentes (ZIMMERMANN, 1996). Como no exemplo apresentado na subseção anterior, o grau de similaridade também será, aqui, definido com base na distância euclidiana entre os elementos, em um espaço de dimensão p.

Como ilustração, no exemplo da subseção acima, a matriz-padrão correspondente aos elementos $\mathrm{x}_{1}, \mathrm{x}_{2}, \ldots, \mathrm{x}_{6}$ possui seis linhas, pois o número de elementos é $\mathrm{n}=6$ sendo cada linha formada pelas componentes que re-

Figura 1 - Subconjuntos $C_{1}$ e $C_{2}$

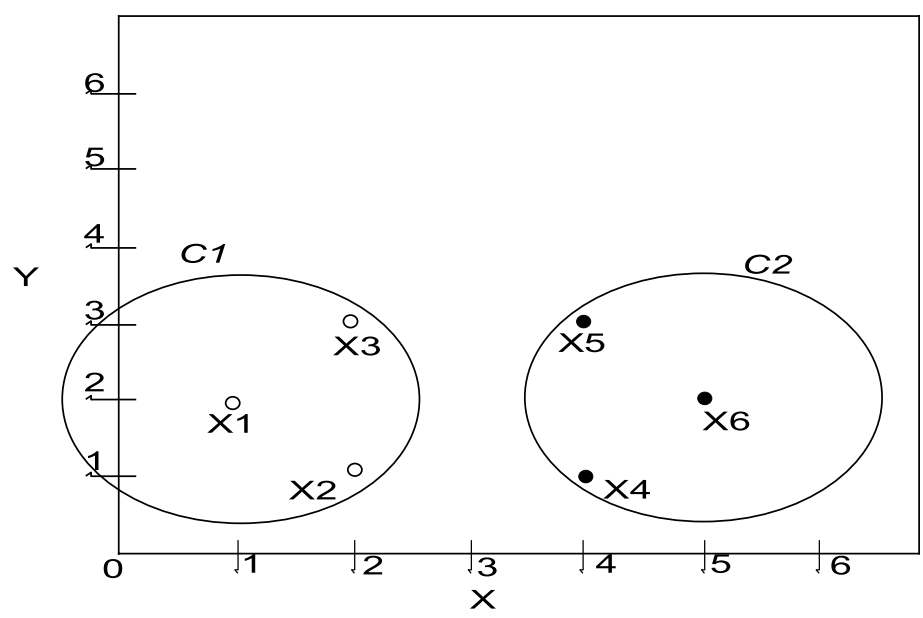


presentam as características dos elementos, ou seja, as coordenadas no espaço de dimensão $\mathrm{p}=2$. Assim, neste exemplo, a matriz-padrão, $\mathrm{M}$, será dada por:

$$
M=\left[\begin{array}{l}
x_{1} \\
x_{2} \\
x_{3} \\
x_{4} \\
x_{5} \\
x_{6}
\end{array}\right]=\left[\begin{array}{ll}
1 & 2 \\
2 & 1 \\
2 & 3 \\
4 & 1 \\
4 & 3 \\
5 & 2
\end{array}\right]
$$

Como na aplicação específica deste trabalho os elementos estudados serão agrupados em dois subgrupos apenas, a apresentação que se segue, descrevendo os conceitos e os passos do algoritmo FCM, será particularizada para essa situação. Assim, sejam $\mathrm{x}_{1}, \mathrm{x}_{2}, \ldots, \mathrm{x}_{\mathrm{m}}$ elementos de um conjunto $\mathrm{X}$, e considere o problema de agrupar estes elementos em dois subgrupos, $\mathrm{C}_{1}$ e $\mathrm{C}_{2}$, de elementos de $\mathrm{X}$. Esses subgrupos devem ser caracterizados através dos índices de pertinência associados aos elementos $\mathrm{x}_{1}$, $\mathrm{x}_{2}, \ldots, \mathrm{x}_{\mathrm{m}}$, de forma que $\mu_{1}\left(\mathrm{x}_{\mathrm{i}}\right)>\mu_{2}\left(\mathrm{x}_{\mathrm{i}}\right)$ para todo $\mathrm{x}_{\mathrm{i}}$ em $\mathrm{C}_{1}$ e $\mu_{2}\left(x_{j}\right)>\mu_{1}\left(x_{j}\right)$ para todo $x_{j}$ em $C_{2}$, ou seja, a pertinência de um elemento de $C_{1}$, relativamente a $C_{1}$, deve ser maior do que a sua pertinência relativamente $a C_{2}$ e a pertinência de um elemento de $C_{2}$, relativamente a $C_{2}$, deve ser maior do que a sua pertinência relativamente a $C_{1}$. Para os casos em que $\mu_{2}\left(\mathrm{x}_{\mathrm{j}}\right)=\mu_{1}\left(\mathrm{x}_{\mathrm{j}}\right)=0,5$, adota-se qualquer das possibilidades $\mathrm{x}_{\mathrm{j}} \in \mathrm{C}_{1}$ ou $\mathrm{x}_{\mathrm{j}} \in \mathrm{C}_{2}$. $\mathrm{O}$ algoritmo $\mathrm{FCM}$ determina os subgrupos $C_{1}$ e $C_{2}$ através da solução do problema seguinte.

Dados os elementos $\mathrm{x}_{1}, \mathrm{x}_{2}, \ldots, \mathrm{x}_{\mathrm{m}}$, descritos na forma de vetores de dimensão $\mathrm{p}$, determinar os vetores $\mathrm{c}_{1} \mathrm{e}$ $c_{2}$, também de dimensão $\mathrm{p}$, e os índices de pertinência $\mu_{1}\left(\mathrm{x}_{\mathrm{j}}\right) \geq 0$ e $\mu_{2}\left(\mathrm{x}_{\mathrm{j}}\right) \geq 0, \mathrm{j}=1,2, \ldots, \mathrm{m}$, satisfazendo $\mu_{1}\left(\mathrm{x}_{\mathrm{j}}\right)+$ $\mu_{2}\left(x_{j}\right)=1, j=1,2, \ldots, m$, de forma a minimizar a função $\sum_{i=1}^{2} \sum_{j=1}^{m}\left[\mu_{i}\left(x_{j}\right)^{2}\left\|x_{j}-c_{i}\right\|^{2}\right]$

Esse problema pode ser escrito de forma abreviada, como o seguinte problema de otimização:

$$
\begin{aligned}
& \min _{\substack{\mu_{i}\left(x_{j},,_{1}, c_{2} \\
i=1,2 \\
j=1, \ldots, m\right.}} \sum_{i=1}^{2} \sum_{j=1}^{m}\left[\mu_{i}\left(x_{j}\right)^{2}\left\|x_{j}-c_{i}\right\|^{2}\right] \\
& \text { sujeito a } \\
& \mu_{1}\left(x_{j}\right)+\mu_{2}\left(x_{j}\right)=1, j=1,2, \ldots, m
\end{aligned}
$$

Em geral, os vetores $c_{1}$ e $c_{2}$, chamados vetores de centro associados aos subgrupos $C_{1}$ e $C_{2}$, não coincidem com nenhum dos elementos de $\mathrm{X}$. Cada vetor de centro carac- teriza um subgrupo como uma concentração de elementos ao seu redor. Esse centro, por sua vez, pode ser visto como uma referência de similaridade entre os elementos. Em outras palavras, o centro associado ao subgrupo pode ser entendido como um padrão que representa todos os elementos que o cercam. Dessa forma, o centro associado ao subgrupo terá sempre um índice de pertinência igual a 1 , relativamente a esse subgrupo, e o índice de pertinência de cada elemento em relação ao subgrupo está associado à distância entre cada elemento e o centro correspondente. Quanto maior a similaridade de um elemento em relação a determinado subgrupo, isto é, quanto menor a distância de um elemento ao centro de determinado subgrupo, mais próximo da unidade será o seu índice de pertinência em relação a esse subgrupo.

O problema de otimização dado pela equação (1) pode ser resolvido analiticamente, e a solução é dada por Bezdek (1981):

$$
\begin{gathered}
c_{i}=\frac{1}{\sum_{j=1}^{m}\left(\mu_{i}\left(x_{j}\right)\right)^{2}} \sum_{j=1}^{m}\left(\mu_{i}\left(x_{j}\right)\right)^{2} x_{j} \quad \mathrm{i}=1,2 \\
\mu_{\mathrm{i}}\left(\mathrm{x}_{\mathrm{j}}\right)=\frac{\frac{1}{\left\|\mathrm{x}_{\mathrm{j}}-\mathrm{c}_{\mathrm{i}}\right\|^{2}}}{\sum_{\mathrm{k}=1}^{\mathrm{m}}\left(\frac{1}{\left\|\mathrm{x}_{\mathrm{k}}-\mathrm{c}_{\mathrm{i}}\right\|^{2}}\right)} \quad \mathrm{i}=1,2 \quad \mathrm{j}=1,2, \ldots, \mathrm{m}
\end{gathered}
$$

Como se pode observar, o cálculo dos vetores de centro, $c_{i}$, pela equação (2), depende dos índices de pertinência, $\mu_{\mathrm{i}}\left(\mathrm{x}_{\mathrm{j}}\right)$. Estes, por sua vez, dependem de $\mathrm{c}_{\mathrm{i}}$, conforme a equação (3). A solução pode ser obtida de forma iterativa, através do algoritmo denominado FCM, cujos passos são descritos a seguir.

Passo 1: Iniciar uma matriz de índices de pertinência, de modo que $\mu_{1}\left(x_{j}\right)+\mu_{2}\left(x_{j}\right)=1, j=1,2, \ldots$, m e $\mu_{1}\left(x_{j}\right) \geq$ 0 e $\mu_{2}\left(x_{\mathrm{j}}\right) \geq 0, \mathrm{j}=1,2, \ldots, \mathrm{m}$;

Passo 2: Calcular os centros $c_{1}$ e $c_{2}$, através da equação (2);

Passo 3: Recalcular, através da equação (3), a nova matriz de pertinências, utilizando os vetores dos centros obtidos no passo 2.

Repetir os passos 2 e 3 até que o valor da função objetivo, equação (1), não mais decresça, segundo a precisão adotada.

Como exemplo, a aplicação do algoritmo FCM ao exemplo 1 da seção 2 produz como resultado os centros $c_{1}=(1,65 ; 1,99)$ e $c_{2}=(4,34 ; 2,00)$ e os índices de 
pertinência mostrados na Tabela 1. Pelos resultados (ver Tabela 1: Índices de pertinência de cada elemento em relação a cada subgrupo), pode-se observar que os centros resultantes não coincidem com nenhum dos elementos dados, o que representa a situação mais freqüente nas aplicações. De fato, a coincidência de elementos com centros caracteriza uma simetria na distribuição dos elementos de cada subgrupo em torno de um dos elementos do subgrupo, o que é improvável quando se utilizam dados reais; ou o elemento que coincide com o centro está muito afastado dos demais elementos do grupo, caracterizando, isoladamente, um subgrupo constituído de um único elemento. Nesse caso, é recomendado eliminar este elemento do conjunto de elementos em estudo (ZIMMERMANN, 1996).

Na seção seguinte, é proposto um novo modelo, baseado em conjuntos Fuzzy, para classificação de ações. Assim como os modelos citados anteriormente, o modelo proposto também incorpora as características de representatividade e ancoramento.

\section{MODELO FUZZY COMPORTAMENTAL}

Nesta seção é apresentada a metodologia utilizada para o desenvolvimento do modelo Fuzzy comportamental proposto. Esse modelo constitui-se de duas etapas de análise: reconhecimento de padrões e classificação de ações. Os dados ou características das ações, utilizados pelo modelo, são indicadores financeiros de companhias abertas, compreendendo índices de rentabilidade, índices de avaliação de ações e um índice de endividamento líquido. Para este trabalho, coletaram-se no banco de dados Economática (2005) índices trimestrais dessa natureza relativos ao período compreendido entre o $4^{\circ}$ trimestre de 1994 e o $3^{\circ}$ trimestre de 2005.

A relação entre esses índices e o retorno financeiro das ações de determinada companhia é um tema bastante discutido na literatura (GITMAN, 1984; CASAGRANDE NETO, SOUSA e ROSSI, 2000; MARTINS, 2001). Para o desenvolvimento deste trabalho foram testados diversos conjuntos de índices financeiros relacionados à liquidez, à rentabilidade, ao endividamento e à avaliação de ações, agrupados de diferentes maneiras. Os índices aqui selecionados foram os que produziram melhores resultados e foram os índices efetivamente adotados. Esses índices dividem-se em:

- Índices de rentabilidade: Giro do Ativo (GA), Margem Líquida (ML), Rentabilidade do Ativo (RA), Rentabilidade do Patrimônio Líquido (RPL) (GITMAN, 1984);

- Índice de endividamento: Endividamento líquido sobre o patrimônio líquido (GITMAN, 1984);

- Índices de avaliação de ações: Preço sobre Valor Patrimonial da Ação (P/VPA), Preço sobre Lucro por Ação (P/L) (MATARAZZO, 1998).

As duas etapas do modelo proposto são descritas a seguir.

Etapa 1: nesta etapa, denominada etapa de reconhecimento de padrões, utilizou-se, para um dado conjunto de ações, o algoritmo FCM para classificação de ações em dois grupos. Conforme visto na segunda seção, cada grupo é representado por um vetor de centro e as ações classificadas nesse grupo serão aquelas com maiores índices de pertinência, quando comparados aos índices relativos ao outro grupo. Essa análise foi baseada em informações trimestrais divulgadas pelas companhias no período que se estende desde o $4^{\circ}$ trimestre de 1994 até o $3^{\circ}$ trimestre de 2000. Em cada trimestre t, o algoritmo FCM foi aplicado à matriz padrão $\mathrm{n} \times \mathrm{p}$, em que cada uma das $\mathrm{n}$ linhas corresponde a uma companhia do grupo estudado e cada uma das p colunas corresponde aos índices financeiros associados à companhia. Como resultado, foram obtidos dois grupos e calculou-se o log-retorno médio que cada grupo produziu ao final do trimestre $t+1$, con-

Tabela 1 - Índices de pertinência de cada elemento em relação a cada subgrupo

\begin{tabular}{|c|c|c|}
\hline ELEMENTO & $\mu_{1}$ & $\mu_{2}$ \\
\hline$x_{1}$ & 0,9630 & 0,0370 \\
\hline$x_{2}$ & 0,8532 & 0,1468 \\
\hline$x_{3}$ & 0,8530 & 0,1470 \\
\hline$x_{4}$ & 0,1470 & 0,8530 \\
\hline$x_{5}$ & 0,1468 & 0,8532 \\
\hline$x_{6}$ & 0,0370 & 0,9630 \\
\hline
\end{tabular}


forme equação (4):

$$
r_{t+1}=\frac{1}{n} \sum_{i=1}^{n} \log \left(\frac{P_{t+1}^{i}}{P_{t}^{i}}\right)
$$

sendo:

$\mathrm{P}_{\mathrm{t}}^{\mathrm{i}}$ - valor da ação i ao final do trimestre $\mathrm{t}$;

$\mathrm{P}_{\mathrm{t}+1}^{\mathrm{i}}$ - valor da ação i ao final do trimestre $\mathrm{t}+1$;

$\mathrm{n}$ - número de ações classificadas no grupo.

O grupo que produziu um retorno financeiro médio maior foi denominado "grupo bom" e o que produziu menor retorno financeiro médio foi denominado "grupo ruim". É interessante observar que, nessa etapa de reconhecimento de padrão, a caracterização do grupo como bom ou ruim só é possível ao final do trimestre $\mathrm{t}+1$, ou seja, a posteriori. O objetivo da etapa 2 é estabelecer um procedimento que permita, já ao final do trimestre $t$, caracterizar o grupo que supostamente terá um desempenho bom ou ruim ao final do trimestre $\mathrm{t}+1$.

Etapa 2: esta etapa, denominada etapa de classificação de ações, foi desenvolvida a partir dos resultados obtidos na etapa 1 e tem como objetivo obter uma classificação, ao final do trimestre $t$, dos grupos de ações com desempenho presumivelmente "bom" e "ruim" ao final do trimestre t +1 . Assim, diferentemente da etapa 1 , pretende-se aqui fazer uma classificação a priori.

Como já descrito, o vetor de centro obtido na etapa 1 para cada grupo é um referencial de semelhança para as ações daquele grupo. Para essa segunda etapa, foram considerados, separadamente, os conjuntos formados pelos centros dos $1^{\text {os }}$ trimestres, $2^{\text {os }}$ trimestres, 3 os trimestres e 4 os trimestres nos anos de 1995 a 2000.

Como ilustração, para os $1^{\text {os }}$ trimestres, há seis $1^{\text {os }}$ trimestres ( $1^{\circ}$ trim./1995, $1^{\circ}$ - trim./1996, $1^{\circ}$ trim./1997, $1^{\circ}$ o trim./1998, 1o trim./1999 e $1^{\circ}$ o trim./2000) e para cada um desses trimestres tem-se um vetor de centro bom e um ruim. Resultam, portanto, 12 vetores de centros, sendo seis bons e seis ruins. Com o objetivo de obter um único vetor de centro bom e um único vetor de centro ruim capazes de representar, respectivamente, os seis centros bons e os seis centros ruins, formou-se uma matriz-padrão composta por esses 12 vetores de centros e, novamente, aplicou-se o algoritmo FCM. Para obter o resultado, determina-se o vetor de centro dos centros bons, aqui denominado vetor de centro vencedor, ao redor do qual os vetores de centros bons se concentram e outro vetor de centro, denominado vetor de centro perdedor, cercado pelos vetores de centros ruins. Os vetores de centro vencedor e perdedor são assumidos, respectivamente, como referenciais para as ações promissoras e não promissoras naquele trimestre. O mes- mo procedimento foi adotado para obtenção dos vetores de centro vencedor e perdedor para os $2^{2 \text { os }}$ trimestres, $3^{\text {os }}$ trimestres e 4 os trimestres.

Para a classificação de uma ação em determinado trimestre, basta calcular os índices de pertinência relativos aos centros vencedor e perdedor correspondentes àquele trimestre. $O$ índice de pertinência maior define a ação como promissora ou não promissora naquele trimestre. No que se segue, para cada trimestre, o grupo de ações promissoras será denominado carteira vencedora e o grupo de ações não promissoras será denominado carteira perdedora. Para os resultados numéricos, a classificação de ações foi feita no período do $1^{\circ}$ trimestre de 2001 ao 3 - trimestre de 2005.

As ações consideradas pertencem ao setor petróleo/ petroquímica, sendo as seguintes: Ciquine, Copene, Copesul, Ipiranga Dist, Ipiranga Pet, Ipiranga Ref, Oxiteno, Petrobras, Petroflex, Petroquímica União, Polialden, Politeno, Química Geral, Supergasbras, Triken, Unipar, e as seguintes ações do setor têxtil: Alpargatas, Alpargatas Santista, Brasperola, Cambuci, Cia. Hering, Coteminas, Cremer, Fab. C. Renaux, Guararapes, Karsten, Marisol, Pettenati, Tecel S. José, Teka.

Ressalta-se que algumas das ações consideradas possuem baixa liquidez e, conseqüentemente, o preço pode não refletir o valor e o contexto atual destas empresas. Embora não tenha sido usado nenhum mecanismo para o tratamento deste fato, tal como a introdução de um fator de ajuste dos preços, acredita-se que procedimentos como esses podem minimizar eventuais distorções nos resultados, devendo, assim, ser objeto de pesquisa futura. Por outro lado, desconsiderar as ações com baixa liquidez não seria adequado devido ao reduzido tamanho da amostra.

A partir das carteiras vencedoras e perdedoras, na seção seguinte são descritos procedimentos para testes das hipóteses de sobre-reação e sub-reação.

\section{TESTES DE HIPÓTESES DE SOBRE-REAÇÃO E SUB- REAÇÃOO}

Os fenômenos de sobre-reação e sub-reação têm sido freqüentemente estudados a partir de pesquisas empíricas (BARBERIS, SHLEIFEER e VISHNY, 1998; DeBONDT e THALER, 1985; KIMURA, 2003; MINARDI, 2004). O fenômeno de sobre-reação caracteriza-se pelo fato de perdedores passados passarem a vencedores futuros, ou vice-versa, e o fenômeno de sub-reação ocorre quando vencedores passados permanecem vencedores no futuro ou perdedores passados permanecem perdedores no 
futuro.

Os procedimentos para a realização dos testes empíricos das hipóteses de sobre-reação e sub-reação, juntamente com os testes de significância estatística, apresentados neste trabalho, são semelhantes àqueles realizados em DeBondt e Thaler (1985), e são descritos a seguir.

i) Utilizando os vetores de centro vencedor e perdedor, ações são classificadas no final de cada trimestre t, como descrito na terceira seção. São assim formadas as carteiras vencedora e perdedora para cada trimestre $\mathrm{t}+1$.

ii) Utilizando as carteiras vencedora e perdedora, obtidas ao final de trimestre $\mathrm{t}$, calcula-se o retorno residual correspondente, para cada semana do trimestre $t+1$, definido conforme as equações (5)-(7),

$$
\begin{aligned}
& \mathrm{RR}_{t+1, j}^{\mathrm{V}}=r_{t+1, j}^{\mathrm{V}}-r_{t+1, j}^{I b b v} \\
& r_{t+1, j}^{\mathrm{V}}=\frac{1}{n} \sum_{\mathrm{i}=1}^{\mathrm{n}} \log \left(\frac{\mathrm{P}_{t+1, j}^{\mathrm{i}}}{\mathrm{P}_{t}^{\mathrm{i}}}\right) \\
& r_{t+1, j}^{\text {Ibov }}=\log \left(\frac{\operatorname{Ibov}_{t+1, j}}{\operatorname{Ibov}_{t}}\right)
\end{aligned}
$$

sendo:

$\mathrm{RR}_{\mathrm{t}+1, \mathrm{j}}^{\mathrm{V}}$ - retorno residual para a carteira vencedora, na semana $\mathrm{j}$ do trimestre $\mathrm{t}+1$

$\mathrm{r}_{\mathrm{t}+1, \mathrm{j}}^{\mathrm{V}}$ - retorno da carteira vencedora, na semana $\mathrm{j}$ do trimestre $\mathrm{t}+1$

$\mathrm{r}_{\mathrm{t}+1, \mathrm{j}}^{\mathrm{rbov}}$ - retorno associado ao Ibovespa, na semana $\mathrm{j}$ do trimestre $\mathrm{t}+1$

$\mathrm{P}_{\mathrm{t}+1, \mathrm{j}}^{\mathrm{i}}$ - valor da ação $\mathrm{i}$, da carteira vencedora, no final da semana $\mathrm{j}$ do trimestre $\mathrm{t}+1$

$\mathrm{P}_{\mathrm{t}}^{\mathrm{i}}$ - valor da ação i, da carteira vencedora, no final do trimestre $\mathrm{t}$

Ibov $_{t+1, j}$ - índice Bovespa no final da semana j do trimestre $\mathrm{t}+1$

$\mathrm{Ibov}_{\mathrm{t}}$ - índice Bovespa no final do trimestre $\mathrm{t}$

$\mathrm{n}$ - número de ações da carteira vencedora

Cálculos análogos são feitos para a carteira perdedora.

iii) Com base nos retornos residuais correspondentes às semanas de cada trimestre, são calculados os retornos residuais médios da carteira vencedora, $R_{R M} \mathrm{t}_{\mathrm{t}}^{\mathrm{V}}$, e da carteira perdedora, $\mathrm{RRM}_{\mathrm{t}}^{\mathrm{P}}$, para cada trimestre, do $1^{\circ}$ trimestre/2001 ao 3o trimestre/2005. A hipótese de sobrereação diz que $R R M_{t}^{V}-R R M_{t}^{P}<0$, visto que o retorno residual médio da carteira perdedora supera o retorno residual médio produzido pela carteira vencedora
(BARBERIS, SHLEIFER e VISHNY, 1998; KIMURA, 2003). A fim de avaliar se, em cada trimestre, a diferença entre os retornos residuais médios é estatisticamente significante, é feito um teste de estatística-t. A hipótese nula a ser testada é $H_{0}: R_{R} M_{t}^{\mathrm{P}}-R_{R} M_{t}^{\mathrm{V}}=0$, contra a hipótese alternativa de sobre-reação $H_{A}$ : $R_{R M} M_{t}^{v}$ $\mathrm{RRM}_{\mathrm{t}}^{\mathrm{P}}<0$. De forma análoga, a hipótese alternativa para sub-reação resulta $\mathrm{H}_{\mathrm{A}}$ : $\mathrm{RRM}_{\mathrm{t}}^{\mathrm{V}}-\mathrm{RRM}_{\mathrm{t}}^{\mathrm{P}}>0$.

\section{RESULTADOS}

Nesta seção o Modelo Fuzzy Comportamental proposto na terceira seção é inicialmente utilizado para a formação de carteiras e, em seguida, são feitos testes para as hipóteses de sobre-reação e sub-reação, utilizando-se a metodologia da quarta seção.

Como exemplo dos resultados obtidos, na Figura 2 são mostrados os gráficos dos retornos residuais para a carteira vencedora e para a carteira perdedora obtidas no final do 3o trimestre de 2002, bem como a diferença entre eles durante cada semana do $4^{\circ}$ trimestre de 2002, para o setor petróleo/petroquímica. Nesse caso, são evidentes os indícios de sobre-reação (ver Figura 2: Retornos residuais semanais durante o $4^{\circ}$ trimestre de 2002 para o setor petróleo/petroquímica).

Calculando os retornos residuais médios para esse trimestre, para as carteiras vencedora e perdedora, constatase que a carteira perdedora supera em aproximadamente $7,85 \%$ o retorno residual médio da carteira vencedora, significante ao nível de 5\% (estatística t: 3,483).

As Tabelas 2 (Retornos residuais médios e Teste-t para o setor petróleo/petroquímica) e 3 (Retornos residuais médios e Teste-t para o setor têxtil) apresentam os resultados obtidos utilizando o Modelo Fuzzy Comportamental, considerando grupos vencedores e grupos perdedores para ativos dos setores de petróleo e petroquímica e de têxtil, respectivamente. Nas tabelas são mostrados os retornos residuais médios para os grupos vencedor e perdedor, bem como a diferença entre o retorno residual médio do grupo vencedor e o retorno residual médio do grupo perdedor. Assim, uma diferença positiva sugere sub-reação e uma diferença negativa sugere sobre-reação. Os t-calculados identificam as significâncias estatísticas das diferenças entre os retornos residuais. Nota-se que na maioria dos trimestres há indícios de sobre-reação para o setor petróleo/petroquímica e sub-reação para o setor têxtil estatisticamente significantes.

Por exemplo, no 3을 trimestre de 2002, para o setor petróleo/petroquímica mostrado na Tabela 2 , a estratégia 
contrária possibilita um ganho de $12,45 \%$, estatisticamente significante. Já para o setor têxtil, no 4o trimestre de 2002, mostrado na Tabela 3, a estratégia de momento possibilita um ganho de $14,67 \%$. Assim, caracteriza-se a influência predominante da heurística de representatividade quando se consideram ações do setor de petróleo/ petroquímica, visto que na maioria dos trimestres ocorre sobre-reação estatisticamente significante (ver Tabela 2: Retornos residuais médios e Teste-t para o setor de petróleo/petroquímica). Por outro lado, a heurística de ancoramento predomina quando se consideram ações do setor têxtil, pois na maioria dos trimestres ocorre sub-reação (ver Tabela 3: Retornos residuais médios e Teste-t para o setor têxtil). Dessa forma, os resultados sugerem que os efeitos de sobre-reação e sub-reação são funções do setor de companhias.

Ressalta-se que, para ações do setor têxtil, em alguns trimestres o retorno residual médio da carteira perdedo$\mathrm{ra}, \mathrm{RRM}_{\mathrm{t}}^{\mathrm{P}}$, é igual a zero. Isso ocorre devido ao fato de todas as ações consideradas terem sido classificadas como vencedoras, ou seja, não houve ações classificadas como perdedoras pelo modelo proposto.

Além disso, como pode ser visto nas Tabelas 2 e 3, nota- se que nos resultados obtidos existe uma alternância entre sub-reação e sobre-reação em alguns trimestres. Sendo assim, as indicações de sobre-reação para o setor de petróleo/ petroquímica e sub-reação para o setor têxtil devem ser vistas como uma informação de apoio ao analista quando da tomada de decisão. Obviamente, a decisão final de investimento deve levar em conta um conjunto mais amplo de informações, tanto quantitativas quanto qualitativas.

\section{CONCLUSÃO}

Neste artigo, é proposto um modelo para a classificação de ações, visando à realização de testes empíricos das hipóteses de sobre-reação e sub-reação no mercado de ações brasileiro, bem como a identificação de viés comportamental nos preços dos ativos financeiros.

O modelo proposto baseia-se na teoria de conjuntos Fuzzy, que por sua vez possui estreita relação com a teoria de finanças comportamentais, sugerindo assim a denominação de Modelo Fuzzy Comportamental. Para a formação de carteiras, o modelo proposto utiliza indicadores financeiros de companhias abertas.

Figura 2 - Retornos residuais semanais durante o 4ํtrimestre de 2002 para 0 setor de petróleo/petroquímica

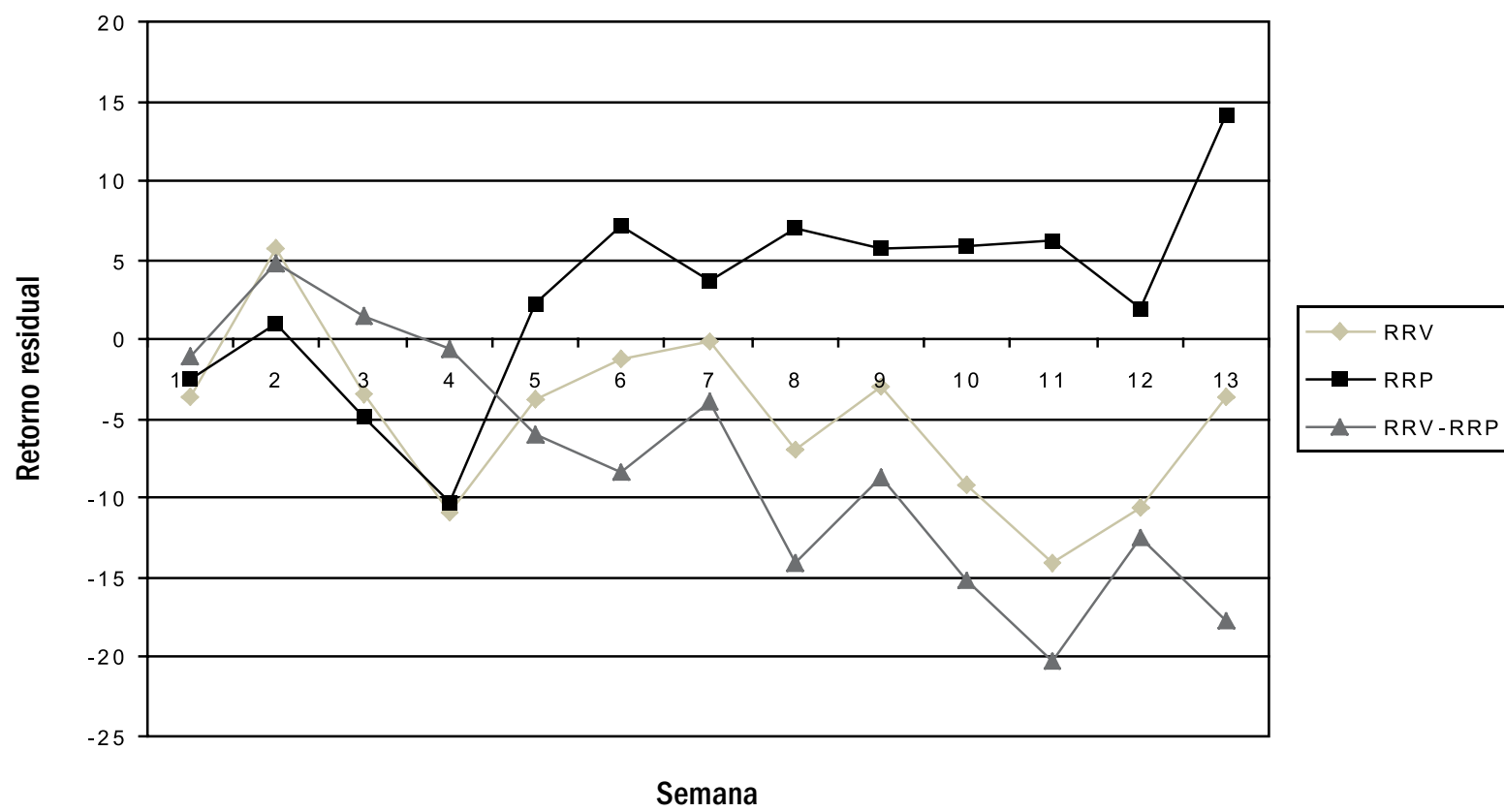


Neste estudo, foram utilizados dois conjuntos de ações: do setor petróleo e petroquímica e do setor têxtil. O setor de petróleo e petroquímica apresenta indícios estatisticamente significantes de sobre-reação, indicando forte influência da heurística de representatividade, segundo a qual é dada mais importância às informações recentes. No caso das carteiras formadas com ações do setor têxtil, constatam-se indícios de sub-reação, que indicam influência significativa da heurística de ancoramento na formação dos grupos, ou seja, excesso de moderação. Assim, é possível através do modelo proposto, investigar o grau de influência das heurísticas de representatividade e ancoramento na tomada decisão em função do setor considerado. Além disso, esses fatores comportamentais têm um relacionamento direto com o modelo aqui proposto nos seguintes sentidos: a heurística de representatividade está baseada principalmente na similaridade, a qual está intimamente relacionada com o grau de pertinência do Modelo Fuzzy Comportamental; por outro lado, a heu- rística de ancoramento está relacionada com a formação dos grupos vencedores e perdedores com base em um referencial para cada grupo, representado pelo vetor de centro, que pode ser visto como uma âncora, com grau de pertinência igual a 1 .

Essas constatações, aliadas ao elo existente entre conjuntos Fuzzy e a teoria de finanças comportamentais, aponta, assim, para influências, associadas a diferentes setores, das heurísticas de representatividade e ancoramento no comportamento do mercado de ações brasileiro.

A importância dos fenômenos de sobre-reação ou subreação decorre do fato de, na tomada de decisão, a sobrereação justificar a escolha da estratégia de investimento denominada estratégia contrária e a sub-reação justificar a escolha da estratégia de momento. Assim, ao adotar-se o modelo proposto para uma situação de tomada de decisão que considere certas ações do setor de petróleo e petroquímica, os resultados sugerem que o investidor poderá realizar um ganho anormal com a compra da carteira per-

Tabela 2 - Retornos residuais médios e Teste-t para o setor de petróleo/petroquímica

\begin{tabular}{|c|c|c|c|c|c|}
\hline TRIMESTRE/ANO & $\operatorname{RRM}_{t}^{v}$ & $\operatorname{RRM}_{t}^{p}$ & $\operatorname{RRM}_{t}^{\mathrm{v}}-\mathrm{RRM}_{t}^{\mathrm{P}}$ & TESTE-t & SOBRE/SUB-REAÇÃO \\
\hline $1^{0}$ trim./2001 & 15,700 & $-2,198$ & 17,898 & $6,232 *$ & Sub \\
\hline $2^{0}$ trim./2001 & 4,449 & $-7,152$ & 11,601 & $4,343^{*}$ & Sub \\
\hline 30 trim./2001 & $-1,759$ & $-14,238$ & 12,479 & $3,022 *$ & Sub \\
\hline 40 trim./2001 & 0,417 & 1,236 & $-0,819$ & $-0,369$ & Sobre \\
\hline 10 trim./2002 & 10,198 & 18,828 & $-8,630$ & $-2,671^{*}$ & Sobre \\
\hline 2o trim./2002 & $-8,240$ & $-0,396$ & $-7,844$ & $-2,208 *$ & Sobre \\
\hline 3o trim./2002 & 1,440 & 13,893 & $-12,453$ & $-2,869 *$ & Sobre \\
\hline 40 trim./2002 & $-4,963$ & 2,888 & $-7,851$ & $-3,483^{*}$ & Sobre \\
\hline 10 trim./2003 & $-13,477$ & $-5,327$ & $-8,150$ & $-1,861 * *$ & Sobre \\
\hline $2^{0}$ trim./2003 & 15,046 & 25,200 & $-10,154$ & $-1,746 * *$ & Sobre \\
\hline 30 trim./2003 & 13,528 & 6,935 & 6,593 & 1,516 & Sub \\
\hline 40 trim./2003 & 1,583 & 6,440 & $-4,857$ & $-1,646$ & Sobre \\
\hline $1^{\circ}$ trim./2004 & 3,441 & 6,138 & $-2,697$ & $-1,312$ & Sobre \\
\hline $2^{0}$ trim./2004 & $-6,273$ & $-6,009$ & $-0,264$ & $-0,075$ & Sobre \\
\hline 30 trim./2004 & 6,173 & 19,125 & $-12,951$ & $-3,044^{*}$ & Sobre \\
\hline $4^{0}$ trim./2004 & 22,277 & 9,204 & 13,073 & $2,751^{*}$ & Sub \\
\hline 10 trim./2005 & $-0,064$ & 0,863 & $-0,928$ & $-0,586$ & Sobre \\
\hline $2^{0}$ trim./2005 & $-5,521$ & $-4,043$ & $-1,478$ & $-1,025$ & Sobre \\
\hline 3o trim./2005 & 2,249 & 5,580 & $-3,330$ & $-1,761 * *$ & Sobre \\
\hline
\end{tabular}

Nota: * Estatisticamente significante ao nível de $5 \%$

** Idem $10 \%$ 
dedora e a venda simultânea, a descoberto (que consiste em vender a carteira vencedora pela cotação atual antes de possuí-la, esperando que a cotação caia, para então recomprar a carteira e auferir lucros na transação) da carteira vencedora, o que caracteriza a estratégia contrária. Já para o setor têxtil, o modelo sugere a compra da carteira vencedora e a venda simultânea, a descoberto, da carteira perdedora, caracterizando, assim, a estratégia de momento.

Ressalta-se que os custos de transação e eventuais restrições para venda a descoberto constituem fatores limitadores da aplicação prática das estratégias contrárias e de momento aqui evidenciadas. Dessa forma, o modelo proposto deve ser visto como uma ferramenta complementar ao conjunto de informações que levarão à decisão de investimento.

Nesse sentido, contrastando com o que se espera na teoria moderna de finanças, os resultados obtidos através do modelo proposto sugerem uma postura não racional no processo de tomada de decisão quando os indivíduos possuem viés dadas as heurísticas de representativida- de e ancoramento. Enquanto a Hipótese de Mercados Eficientes, que constitui um dos blocos construtivos da teoria de finanças modernas, afirma que os preços das ações refletem toda informação disponível, os resultados aqui obtidos indicam que o mercado é informacionalmente ineficiente, visto que há evidências significativas de sobre-reação e sub-reação.

Algumas limitações do modelo proposto devem ser destacadas como motivações para pesquisas futuras: as carteiras são formadas distribuindo-se para cada ação o mesmo valor de capital; o modelo foi aplicado considerando conjuntos de ações de companhias de um mesmo setor; uma conseqüência direta desta limitação é a pequena amostra de dados; outras características, quantitativas, tais como o índice de liquidez da ação ou o índice EV/EBITIDA projetado, ou qualitativas, tal como a avaliação da governança corporativa, associadas às empresas, poderiam ser incluídas no modelo proposto. Além disso, a não-utilização de ajuste do retorno em função do risco torna-se, também, um fator limitador do modelo aqui proposto.

Tabela 3 - Retornos residuais médios e Teste-t para o setor têxtil

\begin{tabular}{|c|c|c|c|c|c|}
\hline TRIMESTRE/ANO & $\operatorname{RRM}_{t}^{v}$ & $\operatorname{RRM}_{t}^{\mathrm{P}}$ & $\operatorname{RRM}_{t}^{v}-\operatorname{RRM}_{t}^{P}$ & TESTE-t & SOBRE/SUB-REAÇÃO \\
\hline 1o trim./2001 & 16,260 & 22,544 & $-6,284$ & $-1,973 * *$ & Sobre \\
\hline $2^{0}$ trim./2001 & 0,100 & $-2,827$ & 2,928 & 1,043 & Sub \\
\hline 30 trim./2001 & $-0,057$ & 0,000 & $-0,057$ & $-0,031$ & Sobre \\
\hline 40 trim./2001 & 6,759 & 0,000 & 6,759 & $4,254^{*}$ & Sub \\
\hline $1^{\circ}$ trim./2002 & 1,735 & 4,122 & $-2,387$ & $-1,094$ & Sobre \\
\hline 20 trim./2002 & 1,111 & $-0,711$ & 1,823 & 0,582 & Sub \\
\hline 3 o trim./2002 & 11,172 & 0,000 & 11,172 & $5,959 *$ & Sub \\
\hline 40 trim./2002 & 13,189 & $-1,481$ & 14,669 & $7,190 *$ & Sub \\
\hline $1^{0}$ trim./2003 & $-2,355$ & $-16,486$ & 14,131 & $4,732 *$ & Sub \\
\hline $2^{0}$ trim./2003 & $-9,177$ & 3,519 & $-12,696$ & $-4,344 *$ & Sobre \\
\hline 3o trim./2003 & 14,174 & 0,000 & 14,174 & $7,226^{*}$ & Sub \\
\hline $4^{0}$ trim./2003 & 1,584 & 0,000 & 1,584 & 1,540 & Sub \\
\hline $1^{0}$ trim./2004 & 2,090 & $-14,636$ & 16,726 & $4,700 *$ & Sub \\
\hline $2^{0}$ trim./2004 & 3,265 & $-4,377$ & 7,642 & $3,328 *$ & Sub \\
\hline 3o trim./2004 & 14,171 & 0,000 & 14,171 & $4,307^{*}$ & Sub \\
\hline 40 trim./2004 & $-0,274$ & 0,000 & $-0,274$ & $-0,229$ & Sobre \\
\hline $1^{0}$ trim./2005 & $-1,305$ & 5,494 & $-6,799$ & $-3,228^{*}$ & Sobre \\
\hline 20 trim./2005 & $-6,783$ & $-16,971$ & 10,188 & $3,352 *$ & Sub \\
\hline 30 trim./2005 & $-1,152$ & 18,379 & $-19,531$ & $-4,518^{*}$ & Sobre \\
\hline
\end{tabular}

Nota: * Estatisticamente significante ao nivel de $5 \%$

** Idem $10 \%$ 


\section{REFERÊNCIAS}

AMIR, E; GANZACH, Y. Overreaction and underreaction in analysts' forecasts. Journal of Economic Behavior \& Organization, v. 37, n. 3, p. 333-347, 1998.

BARBERIS, N; SHLEIFER A; VISHNY, R. A model of investor sentiment. Journal of Financial Economics, v. 49, n. 3, p. 307-343, 1998

BATES, J. H. T; YOUNG, M. P. Applying fuzzy logic to medical decision making in the intensive care unit. American Journal of Respiratory and Critical Care Medicine, v. 167, n. 7, p. 948-952, 2003.

BAYTAS, A; CAKICI, N. Do markets overreact: international evidence. Journal of Banking \& Finance, v. 23, n. 7, p. 1121-1144, 1999.

BENZION U; YAGILJ. Portfolio composition choice: a behavioral approach. The Journal of Behavioral Finance, v. 4, n. 2, p. 85-95, 2003.

BEZDEK, J. C. Pattern recognition with fuzzy objective function algorithm. New York: Plenum, 1981

CASAGRANDE NETO, H; SOUSA, L. A; ROSSI, M. C. Abertura do capital de empresas no Brasil: enfoque prático. 3. ed. São Paulo: Atlas, 2000.

DANIEL, K; HIRSHLEIFER, D; SUBRAHMANYAM, A. Investor psychology and security market seasonality. Journal of Finance, v. 53, n. 6, p. 1839-1886, 1998.

DeBONDT, W. F. M; THALER, R. H. Does the sock market overreact? Journal of Finance, v. 40, n.3, p. 793-807, 1985.

DIAMOND, P; KLOEDEN, P. Metric spaces of fuzzy sets, theory and applications. Singapore: World Scientific, 1994.

DOURRA, H; Siy, P. Investment using technical analysis and fuzzy logic. Fuzzy sets and systems, v. 127, n. 2, p. 221-240, 2002.

ECONOMÁTICA Ltda. Software de apoio a investidores. Disponível em: http://www.economatica.com.br. Acesso em 2005.

FAMA, E. Efficient capital markets: a review of theory and empirical work. Journal of Finance, v. 25, n. 5, p. 383-417, 1970.

FAMA, E. Markets efficiency, long-term returns and behavioral Finance. Journal of Finance Economics, v. 49, n. 3, p. 283-306, 1998.

GITMAN, L. J. Princípios de administração financeira. São Paulo: Harper \& Row do Brasil, 1984.

GRAHAM, I; NEWELL, R. B. Fuzzy adaptive control of a first-order process. Fuzzy Sets and Systems, v. 31, n. 1, p. 47-65, 1989.

HONG, H; STEIN, J. C. A unified theory of underreaction, momentum trading, and overreaction in asset markets. Journal of Finance, v. 54, n. 6 , p. $2143-2184,1999$
JEGADEESH, N. Evidence of predictable behavior of security returns. Journal of Finance, v. 45, n. 3, p. 881-899, 1990.

JEGADEESH, N; TITMAN, S. Returns to buying winners and selling losers: implications for stock market efficiency. Journal of Finance, v. 48, n. 1, p. 65-91, 1993.

KAHNEMAN, D; TVERSKY, A. Prospect theory: an analysis of decisions under risk. Econometrica, 47, n. 2, p. 263-291, 979.

KAHNEMANN, D. Maps of bounded rationality: a perspective on intuitive judgment and choice. Prize Lecture, dec. 2002.

KANG, J; LIU, M; NI, S. X. Contrarian and momentum strategies in the China stock market: 1993-2000. Pacific-Basin Finance Journal, v. 10, n. 3, p. 243-265, 2002.

KAUFMANN, A. Introduction to theory of fuzzy subsets. New York: Academic, 1975.

KIMURA, H. Aspectos comportamentais associados às reações do mercado de capitais. RAE-eletrônica, v. 2, n. 1, p. 1-14, 2003. Disponível em http://www.rae.com.br/eletronica/index.cfm?FuseAction $=$ Artigo $\& I D=188$ $0 \&$ Secao $=2 \%$ C2 $\%$ BAPWC $\&$ Volume $=2 \&$ numero $=1 \& A n o=2003$. Acesso em 15.06.2006

LIMA, M.V. Um estudo sobre finanças comportamentais. RAE-eletronica v. 2, n. 1, p. 1-19, 2003. Disponível em http://www.rae.com.br/eletronica/ index.cfm? FuseAction $=$ Artigo $\& I D=1873 \&$ Secao $=2 \% C 2 \%$ BAPWC $\&$ Volum $\mathrm{e}=2 \&$ numero $=1 \& A n o=2003$. Acesso em 15.06.2006

MARKOWITZ, H. M. Portfolio selection. The Journal of Finance, Chicago: American Finance Association, v. 7, n. 1, p. 77-91, 1952.

MARKOWITZ, H. M. Portfolio selection. New York: John Wiley, 1959.

MARTINS, E. (Org) Avaliação de empresas: da mensuração contábil à econômica. São Paulo: Atlas, 2001.

MATARAZZO, D. Análise financeira de balanços. São Paulo: Atlas, 1998.

MINARDI, A. M. A. F. Retornos passados prevêem retornos futuros. RAEeletrônica, v. 3, n. 2, p. 1-18, jul./dez. 2004. Disponível em http://www. rae.com.br/eletronica/index.cfm? FuseAction $=$ Artigo $\& I D=1738 \& S e c a 0=$ FINANAS2 $\&$ Volume=3\&numero=2\&Ano=2004 . Acesso em 15.06.2006

MODIGLIANI, F; MILLER, M. H.. The cost of capital, corporation finance and the theory of investment. The American Economic Review, Nashville: American Economic Association, v. 48, n. 3, p. 261-297, 1958.

OFFERMAN, T; SONNEMANS, J. What's Causing Overreaction? An experimental investigation of recency and the hot-hand effect. Scandinavian Journal of Economics, v. 106, n. 3, p. 533-553, 2004.

PENNINGS, J. M. E; CANDEL, M; EGELKRAUT, T. A behavioral decision-making modeling approach toward hedging services. The Journal of Behavioral Finance, v. 4, n. 2, p. 71-84, 2003.

PETERS, E. Chaos and order in the capital markets. 2. ed. New York: Wiley, 1996 
PETERS, E. Simple and complex market inefficiencies: integrating efficient markets, behavioral finance, and complexity. The Journal of Behavioral Finance, v. 4, n. 4, p. 225-233, 2003.

RITTER, J. R. Behavioral finance. Pacific-Basin Finance Journal, v. 11, n. 4, p. 429-437, 2003

SHARPE, W. F. Capital asset prices: a theory of market equilibrium under conditions of risk. Journal of Finance, Chicago: American Finance Association, v. 19, n. 3, p. 425-442, 1964.

SHILLER, R. J. From efficient market theory to behavioral finance. Connecticut: Yale University, 2002. Disponível em: http://cowles.econ. yale.edu/. Acesso em 10-2002.

SMITH, V. L. Constructivist and ecological rationality economics. Prize Lecture, dec. 2002.
STRACCA, L. Behavioral finance and asset prices: where do we stand? Journal of Economic Psychology, v. 25, n. 3, p. 373-405, 2004.

WONG, F. S; WANG, P. Z. A stock selection strategy using Fuzzy neural networks. Neurocomputing, v. 2, n. 5, p. 233-242, 1991.

WOOLF, P. J; WANG, Y. A Fuzzy logic approach to analyzing gene expression data. Physiol. Genomics, v. 3, n. 1, p. 9-15, 2000.

ZADEH, L. A. Fuzzy sets. Information and Control, v. 8, n. 3, p.338-353, 1965.

ZADEH, L. A. Outline of a new approach to the analysis of complex systems and decision processes. IEEE Transactions on Systems, Man and Cybernetics Information and Control, v. 3, n. 1, p. 28-44, 1973.

ZIMMERMANN, H. J. Fuzzy set theory and its aplication. Boston: Kluwer Academic, 1996. 